\title{
A note on singular integrals with angular integrability
}

Feng Liu ${ }^{1 *}$ (D)

\section{"Correspondence: \\ liufeng860314@163.com \\ 'College of Mathematics and \\ System Sciences, Shandong \\ University of Science and \\ Technology, Qingdao, P.R. China}

\section{Abstract}

In this note we study the rough singular integral

$$
T_{\Omega} f(x)=\text { p.v. } \int_{\mathbb{R}^{n}} f(x-y) \frac{\Omega(y /|y|)}{|y|^{n}} d y,
$$

where $n \geq 2$ and $\Omega$ is a function in $L \log L\left(S^{n-1}\right)$ with vanishing integral. We prove that $T_{\Omega}$ is bounded on the mixed radial-angular spaces $L_{|x|}^{p} L_{\theta}^{\tilde{P}}\left(\mathbb{R}^{n}\right)$, on the vector-valued mixed radial-angular spaces $L_{|x|}^{p} L_{\theta}^{\tilde{p}}\left(\mathbb{R}^{n}, \ell^{\tilde{p}}\right)$ and on the vector-valued function spaces $L^{p}\left(\mathbb{R}^{n}, \ell^{\tilde{p}}\right)$ if $1<\tilde{p} \leq p<\tilde{p} n /(n-1)$ or $\tilde{p} n /(\tilde{p}+n-1)<p \leq \tilde{p}<\infty$. The same conclusions hold for the well-known Riesz transforms and directional Hilbert transforms. It should be pointed out that our proof is based on the Calderón-Zygmund's rotation method.

MSC: Primary 42B20; secondary 42B25

Keywords: Singular integrals; Rough kernels; Directional Hilbert transforms; Riesz transforms; Mixed radial-angular spaces

\section{Introduction}

Singular integral theory was initiated in the seminal work of Calderón and Zygmund [1] and since then has been an active area of research. A celebrated work was due to Calderón and Zygmund [2] who first studied the rough singular integral

$$
T_{\Omega} f(x)=\text { p.v. } \int_{\mathbb{R}^{n}} f(x-y) \frac{\Omega(y /|y|)}{|y|^{n}} d y,
$$

where $\Omega$ is a function in $L \log L\left(\mathrm{~S}^{n-1}\right)$ with vanishing integral,

$$
\int_{S^{n-1}} \Omega(y) d \sigma(y)=0
$$

where $S^{n-1}$ denotes the unit sphere in $\mathbb{R}^{n}(n \geq 2)$ equipped with the normalized Lebesgue measure $d \sigma$. By introducing the "method of rotations", Calderón and Zygmund [2] showed that $T_{\Omega}$ is bounded on the Lebesgue spaces $L^{p}\left(\mathbb{R}^{n}\right)$ for $1<p<\infty$. Here the function class $L \log L\left(\mathrm{~S}^{n-1}\right)$ denotes the set of all functions $\Omega: S^{n-1} \rightarrow \mathbb{R}$ which satisfy

$$
\|\Omega\|_{L \log L\left(S^{n-1}\right)}:=\int_{S^{n-1}}|\Omega(\theta)| \log (2+|\Omega(\theta)|) d \sigma(\theta)<\infty .
$$

(c) The Author(s) 2019. This article is distributed under the terms of the Creative Commons Attribution 4.0 International License (http://creativecommons.org/licenses/by/4.0/), which permits unrestricted use, distribution, and reproduction in any medium, provided you give appropriate credit to the original author(s) and the source, provide a link to the Creative Commons license, and indicate if changes were made. 
The same conclusion was obtained independently by Coifman and Weiss [3] and Connett [4] under the less restrictive condition that $\Omega$ lies in the Hardy space $H^{1}\left(\mathrm{~S}^{n-1}\right)$. The weak type $(1,1)$ bounds of $T_{\Omega}$ were proved by many authors under the condition that $\Omega \in L \log L\left(\mathrm{~S}^{n-1}\right)$ (see [5, 6]). For other developments on this topic we can consult [7-15], among others.

It is well known that the mixed radial-angular space $L_{|x|}^{p} L_{\theta}^{\tilde{p}}\left(\mathbb{R}^{n}\right)$ is merely a formal extension of the Lebesgue space $L^{p}$, but over the last several years it has been successfully used in studying Strichartz estimates and dispersive equations (see [16-28]). Recall that the mixed radial-angular spaces $L_{|x|}^{p} L_{\theta}^{\tilde{p}}\left(\mathbb{R}^{n}\right), 1 \leq p, \tilde{p} \leq \infty$, consist of all functions $u$ satisfying $\|u\|_{L_{|x|}^{p} L_{\theta}^{\tilde{p}}\left(\mathbb{R}^{n}\right)}<\infty$, where

$$
\begin{aligned}
& \|u\|_{L_{|x|}^{p} \tilde{L}_{\theta}^{\tilde{p}}\left(\mathbb{R}^{n}\right)}:=\left(\int_{0}^{\infty}\|u(\rho \cdot)\|_{L^{\tilde{p}}\left(S^{n-1}\right)}^{p} \rho^{n-1} d \rho\right)^{1 / p} \text { and } \\
& \|u\|_{L_{|x|}^{\infty} \tilde{L}_{\theta}^{\tilde{L}}\left(\mathbb{R}^{n}\right)}:=\sup _{\rho>0}\|u(\rho \cdot)\|_{L^{\tilde{p}}\left(S^{n-1}\right)} .
\end{aligned}
$$

It is clear that the spaces $L_{|x|}^{p} L_{\theta}^{\tilde{p}}\left(\mathbb{R}^{n}\right)$ have the following easy properties.

(i) If $p=\tilde{p}$ and $1 \leq p \leq \infty$, then

$$
\|u\|_{L_{|x|}^{p} L_{\theta}^{\tilde{p}}\left(\mathbb{R}^{n}\right)}=\|u\|_{L^{p}\left(\mathbb{R}^{n}\right)}
$$

(ii) If $u$ is a radial function on $\mathbb{R}^{n}$ and $1 \leq p, \tilde{p} \leq \infty$, then

$$
\|u\|_{L_{|x|}^{p} L_{\theta}^{\tilde{p}}\left(\mathbb{R}^{n}\right)} \simeq\|u\|_{L^{p}\left(\mathbb{R}^{n}\right)}
$$

(iii) If $1 \leq \tilde{p}_{1} \leq \tilde{p}_{2} \leq \infty$ and $1 \leq p \leq \infty$, then

$$
\|u\|_{L_{|x|}^{p} L_{\theta}^{\tilde{p}_{1}}\left(\mathbb{R}^{n}\right)} \leq C_{n, p, \tilde{p}_{1}, \tilde{p}_{2}}\|u\|_{\left.L_{|x|}^{p}\right|_{\theta} ^{\tilde{p}_{2}}\left(\mathbb{R}^{n}\right)}
$$

Here the notation $A \simeq B$ means that there are two positive constants $C, C^{\prime}$ such that $A \leq$ $C B$ and $B \leq C^{\prime} A$.

Recently the mixed radial-angular spaces also played an active role in singular integral theory. A good start in this direction was due to Córdoba [29] who proved that $T_{\Omega}$ is bounded on $L_{|x|}^{p} L_{\theta}^{2}\left(\mathbb{R}^{n}\right)$ for all $1<p<\infty$, provided that $\Omega \in \mathcal{C}^{1}\left(\mathrm{~S}^{n-1}\right)$. Later on, D'Ancona and Lucà [30] used the same argument in [29, Theorem 2.1] to extend the above result to the following.

Theorem A ([30]) Let $\Omega \in \mathcal{C}^{1}\left(\mathrm{~S}^{n-1}\right)$ satisfy $(1.2)$ and $1<p, \tilde{p}<\infty$. Then

$$
\left\|T_{\Omega} f\right\|_{L_{|x|}^{p} L_{\theta}^{\tilde{p}}\left(\mathbb{R}^{n}\right)} \leq C_{\Omega, p, \tilde{p}}\|f\|_{L_{|x|}^{p} L_{\theta}^{\tilde{p}}\left(\mathbb{R}^{n}\right)}
$$

Very recently, Cacciafesta and Lucà [31] extended Theorem A to the weighted setting (see [31, Theorem 1.1]). It should be pointed out that

$$
\mathcal{C}^{1}\left(\mathrm{~S}^{n-1}\right) \subsetneq L \log L\left(\mathrm{~S}^{n-1}\right) \subsetneq H^{1}\left(\mathrm{~S}^{n-1}\right) \subsetneq L^{1}\left(\mathrm{~S}^{n-1}\right) .
$$


The main focus of the current note is to consider the $L_{|x|}^{p} L_{\theta}^{\tilde{p}}\left(\mathbb{R}^{n}\right)$ boundedness of $T_{\Omega}$ without assuming that $\Omega$ is in $\mathcal{C}^{1}\left(\mathrm{~S}^{n-1}\right)$ with mean value zero. Actually, we want to improve Theorem A to $\Omega \in L \log L\left(\mathrm{~S}^{n-1}\right)$. To be more precisely, our main result can be formulated as follows.

Theorem 1.1 Let $\Omega \in L \log L\left(\mathrm{~S}^{n-1}\right)$ and satisfy (1.2). If $1<\tilde{p} \leq p<\tilde{p} n /(n-1)$ or $\tilde{p} n /(\tilde{p}+$ $n-1)<p \leq \tilde{p}<\infty$, then the following are valid:

$$
\begin{aligned}
& \left\|T_{\Omega}\right\|_{L_{|x|}^{p} L_{\theta}^{\tilde{p}}\left(\mathbb{R}^{n)}\right.} \leq C_{p, \tilde{p}, \Omega}\|f\|_{\left.L_{|x|}^{p}\right|_{\theta} ^{p}\left(\mathbb{R}^{n}\right)^{n}} ; \\
& \left\|\left(\sum_{j \in \mathbb{Z}}\left|T_{\Omega} f_{j}\right|^{\tilde{p}}\right)^{1 / \tilde{p}}\right\|_{L_{|x|}^{p} L_{\theta}^{\tilde{p}}\left(\mathbb{R}^{n)}\right.} \leq C_{p, \tilde{p}, \Omega}\left\|\left(\sum_{j \in \mathbb{Z}}\left|f_{j}\right|^{\tilde{p}}\right)^{1 / \tilde{p}}\right\|_{\left.L_{|x|}^{p}\right|_{\theta} ^{\tilde{L}^{p}}\left(\mathbb{R}^{n}\right)} ; \\
& \left\|\left(\sum_{j \in \mathbb{Z}}\left|T_{\Omega} f_{j}\right|^{\tilde{p}}\right)^{1 / \tilde{p}}\right\|_{L^{p}\left(\mathbb{R}^{n}\right)} \leq C_{p, \tilde{p}, \Omega}\left\|\left(\sum_{j \in \mathbb{Z}}\left|f_{j}\right|^{\tilde{p}}\right)^{1 / \tilde{p}}\right\|_{L^{p}\left(\mathbb{R}^{n}\right)} .
\end{aligned}
$$

We would like to remark that Theorem 1.1 is based on the Calderón-Zygmund rotation method. In order to prove Theorem 1.1, let us introduce the direction Hilbert transforms and Riesz transforms. For a $w \in \mathbb{R}^{n}$, we define the directional Hilbert transform $\mathcal{H}_{w}$ in the direction $w$ as

$$
\mathcal{H}_{w} f(x)=\frac{1}{\pi} \text { p.v. } \int_{-\infty}^{\infty} f(x-t w) \frac{d t}{t},
$$

where $f \in \mathcal{S}\left(\mathbb{R}^{n}\right)$ (the Schwartz class on $\mathbb{R}^{n}$ ). For $1 \leq j \leq n$, the $j$ th Riesz transform is given by

$$
\mathcal{R}_{j} f(x)=\frac{\Gamma\left(\frac{n+1}{2}\right)}{\pi^{\frac{n+1}{2}}} \text { p.v. } \int_{\mathbb{R}^{n}} f(x-y) \frac{y_{j}}{|y|^{n}} d y,
$$

where $f \in \mathcal{S}\left(\mathbb{R}^{n}\right)$.

Recently, Córdoba [29] proved the following.

Theorem B ([29]) Let $w \in S^{n-1}$. Then $\mathcal{H}_{w}$ is bounded on $L_{|x|}^{p} L_{\theta}^{2}\left(\mathbb{R}^{n}\right)$ if and only if $2 n /(n+1)<p<2 n /(n-1)$.

In this paper we shall extend Theorem B to the following.

Theorem 1.2 Let $w \in \mathrm{S}^{n-1}$. Then $\mathcal{H}_{w}$ defined as (1.8) is bounded on $L_{|x|}^{p} L_{\theta}^{\tilde{p}}\left(\mathbb{R}^{n}\right)$ if and only if $1<\tilde{p} \leq p<\tilde{p} n /(n-1)$ or $\tilde{p} n /(\tilde{p}+n-1)<p \leq \tilde{p}<\infty$. Moreover, the following are valid:

$$
\begin{aligned}
& \left\|\mathcal{H}_{w} f\right\|_{\left.L_{|x|}^{p}\right|_{\theta} ^{\tilde{p}}\left(\mathbb{R}^{n}\right)} \leq C_{p, \tilde{p}}\|f\|_{\left.L_{|x|}^{p}\right|_{\theta} ^{\tilde{L}_{\theta}}\left(\mathbb{R}^{n}\right)^{n}} \\
& \left\|\left(\sum_{j \in \mathbb{Z}}\left|\mathcal{H}_{w} f_{j}\right|^{\tilde{p}}\right)^{1 / \tilde{p}}\right\|_{\left.L_{|x|}^{p}\right|_{\theta} ^{\tilde{L}}\left(\mathbb{R}^{n}\right)} \leq C_{p, \tilde{p}}\left\|\left(\sum_{j \in \mathbb{Z}}\left|f_{j}\right|^{\tilde{p}}\right)^{1 / \tilde{p}}\right\|_{L_{|x|}^{p} L_{\theta}^{\tilde{p}}\left(\mathbb{R}^{n}\right)}, \\
& \left\|\left(\sum_{j \in \mathbb{Z}}\left|\mathcal{H}_{w} f_{j}\right|^{\tilde{p}}\right)^{1 / \tilde{p}}\right\|_{L^{p}\left(\mathbb{R}^{n}\right)} \leq C_{p, \tilde{p}}\left\|\left(\sum_{j \in \mathbb{Z}}\left|f_{j}\right|^{\tilde{p}}\right)^{1 / \tilde{p}}\right\|_{L^{p}\left(\mathbb{R}^{n}\right)} .
\end{aligned}
$$

The above constants $C_{p, \tilde{p}}$ are independent of $w$. 
Theorem 1.2 together with the rotation method yields the following.

Theorem 1.3 Let $\Omega$ be odd and integrable over $S^{n-1}$. If $1<\tilde{p} \leq p<\tilde{p} n /(n-1)$ or $\tilde{p} n /(\tilde{p}+$ $n-1)<p \leq \tilde{p}<\infty$, then the following are valid:

$$
\begin{aligned}
& \left\|T_{\Omega}\right\|_{L_{|x|}^{p} L_{\theta}^{\tilde{p}}\left(\mathbb{R}^{n}\right)} \leq C_{p, \tilde{p}}\|\Omega\|_{L^{1}\left(S^{n-1}\right)}\|f\|_{L_{|x|}^{p} L_{\theta}^{\tilde{p}}\left(\mathbb{R}^{n}\right)} ; \\
& \left\|\left(\sum_{j \in \mathbb{Z}}\left|T_{\Omega} f_{j}\right|^{\tilde{p}}\right)^{1 / \tilde{p}}\right\|_{L_{|x|}^{p} L_{\theta}^{\tilde{p}}\left(\mathbb{R}^{n)}\right.} \leq C_{p, \tilde{p}}\|\Omega\|_{L^{1}\left(S^{n-1}\right)}\left\|\left(\sum_{j \in \mathbb{Z}}\left|f_{j}\right|^{\tilde{p}}\right)^{1 / \tilde{p}}\right\|_{\left.\left.L_{|x|}^{p}\right|_{\dot{\theta}} ^{\tilde{p}} \mathbb{R}^{n}\right)^{n}}, \\
& \left\|\left(\sum_{j \in \mathbb{Z}}\left|T_{\Omega} f_{j}\right|^{\tilde{p}}\right)^{1 / \tilde{p}}\right\|_{L^{p}\left(\mathbb{R}^{n)}\right.} \leq C_{p, \tilde{p}}\|\Omega\|_{L^{1}\left(S^{n-1}\right)}\left\|\left(\sum_{j \in \mathbb{Z}}\left|f_{j}\right|^{\tilde{p}}\right)^{1 / \tilde{p}}\right\|_{L^{p}\left(\mathbb{R}^{n}\right)} .
\end{aligned}
$$

Here the above constants $C_{p, \tilde{p}}>0$ are independent of $\Omega$. The same conclusions hold for the Riesz transforms $\mathcal{R}_{j}$ for all $1 \leq j \leq n$.

Remark 1.1 When $\tilde{p}=2$, the part result of Theorem 1.2 implies Theorem A. On the other hand, Córdoba [29] proved the following, Meyer's lemma: Given a countable family of directions $\left\{\theta_{j}\right\}_{j \in \mathbb{Z}}$ in $\mathbb{R}^{n}$ and set $\mathcal{H}_{j} f=\mathcal{H}_{\theta_{j}} f$. Then the following inequality holds:

$$
\left\|\left(\sum_{j \in \mathbb{Z}}\left|H_{j} f_{j}\right|^{2}\right)^{1 / 2}\right\|_{L_{|x|}^{p} L_{\theta}^{2}\left(\mathbb{R}^{n}\right)} \leq C_{p}\left\|\left(\sum_{j \in \mathbb{Z}}\left|f_{j}\right|^{2}\right)^{1 / 2}\right\|_{L_{|x|}^{p} L_{\theta}^{2}\left(\mathbb{R}^{n}\right)}
$$

for $2 n /(n+1)<p<2 n /(n-1)$. By using the arguments as in deriving (1.11) and (1.12), we find that if $1<\tilde{p} \leq p<\tilde{p} n /(n-1)$ or $\tilde{p} n /(\tilde{p}+n-1)<p \leq \tilde{p}<\infty$, the following inequalities hold:

$$
\begin{aligned}
& \left\|\left(\sum_{j \in \mathbb{Z}}\left|\mathcal{H}_{j} f_{j}\right|^{\tilde{p}}\right)^{1 / \tilde{p}}\right\|_{L_{|x|}^{p} L_{\theta}^{\tilde{p}}\left(\mathbb{R}^{n}\right)} \leq C_{p, \tilde{p}}\left\|\left(\sum_{j \in \mathbb{Z}}\left|f_{j}\right|^{\tilde{p}}\right)^{1 / \tilde{p}}\right\|_{L_{|x|}^{p} L_{\theta}^{\tilde{p}_{\theta}}\left(\mathbb{R}^{n}\right)}, \\
& \left\|\left(\sum_{j \in \mathbb{Z}}\left|\mathcal{H}_{j} f_{j}\right|^{\tilde{p}}\right)^{1 / \tilde{p}}\right\|_{L^{p}\left(\mathbb{R}^{n}\right)} \leq C_{p, \tilde{p}}\left\|\left(\sum_{j \in \mathbb{Z}}\left|f_{j}\right|^{\tilde{p}}\right)^{1 / \tilde{p}}\right\|_{L^{p}\left(\mathbb{R}^{n}\right)} .
\end{aligned}
$$

It is clear that (1.17) yields (1.16) when $\tilde{p}=2$.

Throughout the paper, we use $C_{\alpha, \beta, . .}$ to denote positive constants that depend on the parameters $\alpha, \beta, \ldots$.

\section{Proofs of main results}

Let us begin with the proof of Theorem 1.2.

Proof of Theorem 1.2 We only prove (1.10) since (1.11) and (1.12) are analogous. We shall adopt the method of deriving the proof in [30, Theorem 2.6] to prove (1.8). Let $1<\tilde{p}<$ $p<\tilde{p} n /(n-1)$ and $t=p /(p-\tilde{p})$. It is obvious that $t>n$. Fix a number $s$ in the interval $(1, t / n)$. Denote by $X$ the set of all $g \in \mathcal{S}(\mathbb{R})$ with $\int_{0}^{\infty} g^{t}(r) r^{n-1} d r \leq 1$. By polar coordinates, 
we have

$$
\begin{aligned}
\left\|\mathcal{H}_{w}\right\|_{L_{|x|}^{p} L_{\theta}^{\tilde{p}}\left(\mathbb{R}^{n}\right)}^{\tilde{n}} & =\left(\int_{0}^{\infty}\left(\int_{S^{n-1}}\left|\mathcal{H}_{w} f(r \theta)\right|^{\tilde{p}} d \sigma(\theta)\right)^{p / \tilde{p}} r^{n-1} d r\right)^{\tilde{p} / p} \\
& =\sup _{g \in X} \int_{0}^{\infty} \int_{S^{n-1}}\left|\mathcal{H}_{w} f(r \theta)\right|^{\tilde{p}} g(r) r^{n-1} d \sigma(\theta) d r \\
& =\sup _{g \in X} \int_{\mathbb{R}^{n}}\left|\mathcal{H}_{w} f(x)\right|^{\tilde{p}} g(|x|) d x .
\end{aligned}
$$

Fix $g \in X$ and set $h(x)=g(|x|)$. It is well known that

$$
\begin{aligned}
\int_{\mathbb{R}^{n}}\left|\mathcal{H}_{w} f(x)\right|^{\tilde{p}} g(|x|) d x & \leq C_{\tilde{p}, s} \int_{\mathbb{R}^{n}}|f(x)|^{\tilde{p}}\left(\mathcal{M}_{w} h^{s}(x)\right)^{1 / s} d x \\
& \leq C_{\tilde{p}, s} \int_{\mathbb{R}^{n}}|f(x)|^{\tilde{p}}\left(\mathcal{U} h^{s}(x)\right)^{1 / s} d x
\end{aligned}
$$

for all $p \in(1, \infty)$ and $s \in(1, \infty)$. Here $\mathcal{M}_{w}$ denotes the one-dimensional Hardy-Littlewood maximal function in the direction of $w$ and $\mathcal{U}$ is the universal Kakeya maximal function defined by

$$
\mathcal{U} f(x)=\sup _{\substack{a, b>0 \\ w \in S^{n-1}}} \frac{1}{a+b} \int_{-a}^{b}|f(x+t w)| d t .
$$

It was shown in [32] (also see [29]) that if $f$ is a radial function, then

$$
\|\mathcal{U} f\|_{L^{v}\left(\mathbb{R}^{n}\right)} \leq C_{v}\|f\|_{L^{v}\left(\mathbb{R}^{n}\right)}, \quad \text { for } v>n .
$$

Notice that $t / s>n$ and $h^{s}$ is a radial function. It follows from (2.2)-(2.3) that

$$
\begin{aligned}
& \int_{\mathbb{R}^{n}}\left|\mathcal{H}_{w} f(x)\right|^{\tilde{p}} g(|x|) d x \\
& \quad \leq C_{\tilde{p}, s} \int_{0}^{\infty} \int_{S^{n-1}}|f(r \theta)|^{\tilde{p}} d \sigma(\theta)\left(\mathcal{U} h^{s}(r)\right)^{1 / s} r^{n-1} d r \\
& \left.\quad \leq C_{\tilde{p}, s} \int_{0}^{\infty}\left(\int_{S^{n-1}}|f(r \theta)|^{\tilde{p}} d \sigma(\theta)\right)^{p / \tilde{p}} r^{n-1} d \rho\right)^{\tilde{p} / p}\left(\int_{0}^{\infty}\left(\mathcal{U} h^{s}(r)\right)^{t / s} r^{n-1} d r\right)^{1 / t} \\
& \leq C_{\tilde{p}, s}\|f\|_{L_{|x|}^{p} L_{\theta}^{\tilde{p}}\left(\mathbb{R}^{n}\right)}^{\tilde{p}}\left\|\left(\mathcal{U} h^{s}\right)^{1 / s}\right\|_{L^{t}\left(\mathbb{R}^{n}\right)} \\
& \leq C_{p, \tilde{p}, s} \mid f \|_{\left.L_{|x|}^{p} L_{\theta}^{\tilde{p}} \mathbb{R}^{n}\right)^{n}}
\end{aligned}
$$

which together with (2.1) implies that (1.8) holds for $1<\tilde{p}<p<\tilde{p} n /(n-1)$. By the duality we get the case $\tilde{p} n /(\tilde{p}+n-1)<p<\tilde{p}<\infty$. The trivial case $1<p=\tilde{p}<\infty$ follows easily from the $L^{p}$ bounds for $\mathcal{H}_{w}$ and (1.3).

To prove the "only if" part we take $f=\chi_{B(0,1)}$, where $B(0,1)$ is the unit cube in $\mathbb{R}^{n}$. Without loss of generality we only consider the case $w=(0,0, \ldots, 0,1)$ because of the rotational symmetry. One can easily check that

$$
\left|\mathcal{H}_{w} f\left(x_{1}, x_{2}, \ldots, x_{n-1}, x_{n}\right)\right| \geq C \frac{1}{\left|x_{n}\right|},
$$


whenever $\left|x_{i}\right| \leq \frac{1}{2}, i=1,2, \ldots, n-1$ and $\left|x_{n}\right| \geq 2$. An elementary computation yields

$$
\begin{aligned}
& \int_{0}^{\infty}\left(\int_{S^{n-1}}\left|\mathcal{H}_{w} f(r \theta)\right|^{\tilde{p}} d \sigma(\theta)\right)^{p / \tilde{p}} r^{n-1} d r \\
& \quad \geq C \int_{2}^{\infty}\left(r^{-\tilde{p}} r^{-(n-1)}\right)^{p / \tilde{p}} r^{n-1} d r \geq C \int_{2}^{\infty} r^{(n-1)(1-p / \tilde{p})-p} d r
\end{aligned}
$$

which together with the $L_{|x|}^{p} L_{\theta}^{\tilde{p}}\left(\mathbb{R}^{n}\right)$ boundedness for $\mathcal{H}_{w}$ yields $\tilde{p} n /(\tilde{p}+n-1)<p \leq \tilde{p}<\infty$. The case $1<\tilde{p} \leq p<\tilde{p} n /(n-1)$ follows by duality.

Proof of Theorem 1.3 We shall prove (1.13) and (1.14)-(1.15) are analogous. By the method of rotations, it was shown in [33] that

$$
T_{\Omega} f(x)=\frac{\pi}{2} \int_{S^{n-1}} \Omega(w) \mathcal{H}_{w} f(x) d \sigma(w) .
$$

By (2.4) and Minkowski's inequalities, one has

$$
\begin{aligned}
\left\|T_{\Omega} f\right\|_{L_{|x|}^{p} L_{\theta}^{\tilde{p}}\left(\mathbb{R}^{n}\right)} & \left(\int_{0}^{\infty}\left(\int_{S^{n-1}}\left|T_{\Omega} f(r \theta)\right|^{\tilde{p}} d \sigma(\theta)\right)^{p / \tilde{p}} r^{n-1} d r\right)^{1 / p} \\
\quad= & \frac{\pi}{2}\left(\int_{0}^{\infty}\left(\int_{S^{n-1}}\left|\int_{S^{n-1}} \Omega(w) \mathcal{H}_{w} f(r \theta) d \sigma(w)\right|^{\tilde{p}} d \sigma(\theta)\right)^{p / \tilde{p}} r^{n-1} d r\right)^{1 / p} \\
& \leq \frac{\pi}{2}\left(\int_{0}^{\infty}\left(\int_{S^{n-1}}|\Omega(w)|\left(\int_{S^{n-1}}\left|\mathcal{H}_{w} f(r \theta)\right|^{\tilde{p}} d \sigma(\theta)\right)^{1 / \tilde{p}} d \sigma(w)\right)^{p} r^{n-1} d r\right)^{1 / p} \\
& \leq \frac{\pi}{2} \int_{S^{n-1}}|\Omega(w)|\left(\int_{0}^{\infty}\left(\int_{S^{n-1}}\left|\mathcal{H}_{w} f(r \theta)\right|^{\tilde{p}} d \sigma(\theta)\right)^{p / \tilde{p}} r^{n-1} d r\right)^{1 / p} d \sigma(w) \\
& \leq C_{p, \tilde{p}}\|\Omega\|_{L^{1}\left(S^{n-1}\right)}\|f\|_{L_{|x|}^{p} L_{\theta} \tilde{p}_{\theta}\left(\mathbb{R}^{n}\right)}
\end{aligned}
$$

if $1<\tilde{p} \leq p<\tilde{p} n /(n-1)$ or $\tilde{p} n /(\tilde{p}+n-1)<p \leq \tilde{p}<\infty$. This finishes the proof of Theorem 1.3.

Proof of Theorem 1.1 We first prove that the conclusions of Theorem 1.1 hold for $T_{\Omega}$ if $\Omega$ is an even function and $\Omega \in L \log L\left(\mathrm{~S}^{n-1}\right)$ satisfies (1.2). By [33, Proposition 4.1.16], we obtain

$$
T_{\Omega}=-\sum_{j=1}^{n} \mathcal{R}_{j} \mathcal{R}_{j} T_{\Omega}
$$

where $\mathcal{R}_{j}$ is the $j$ th Riesz transform defined by (1.9). Let $T_{j}=\mathcal{R}_{j} T_{\Omega}$. Fix $1 \leq j \leq n$. By the idea in Grafakos's book [33, pp. 274-278], we see that there exists an odd integrable kernel $\Omega_{j}$ such that

$$
T_{j}=T_{\Omega_{j}}
$$


We get from (2.5) and (2.6)

$$
T_{\Omega}=-\sum_{j=1}^{n} R_{j} T_{\Omega_{j}}
$$

Then (1.5)-(1.7) follow easily from Theorem 1.3 and (2.7).

Let $\Omega$ be given as in Theorem 1.1. We can simply write $\Omega=\Omega_{e}+\Omega_{o}$, where $\Omega_{e}(x)=$ $\frac{\Omega(x)+\Omega(-x)}{2}$ and $\Omega_{o}(x)=\frac{\Omega(x)-\Omega(-x)}{2}$. Then $T_{\Omega}$ can be written as $T_{\Omega}=T_{\Omega_{e}}+T_{\Omega_{0}}$. One can easily check that $\Omega_{e}$ is even and $\Omega_{e} \in L \log L\left(\mathrm{~S}^{n-1}\right)$ satisfies (1.2). $\Omega_{o}$ is odd and $\Omega_{o} \in L^{1}\left(\mathrm{~S}^{n-1}\right)$. Applying the proved claim for $T_{\Omega}$ with even kernel $\Omega$ and Theorem 1.3, we get (1.5)(1.7).

\section{Funding}

The work was supported partially by the NNSF of China (No. 11701333) and Support Program for Outstanding Young Scientific and Technological Top-notch Talents of College of Mathematics and Systems Science (No. Sxy2016K01).

\section{Competing interests}

The author declares to have no competing interests.

\section{Authors' contributions}

The author was the only one to work in drafting and approving the final manuscript.

\section{Publisher's Note}

Springer Nature remains neutral with regard to jurisdictional claims in published maps and institutional affiliations.

Received: 25 April 2019 Accepted: 9 October 2019 Published online: 16 October 2019

\section{References}

1. Calderón, A.P., Zygmund, A.: On the existence of certain singular integrals. Acta Math. 88, 85-139 (1952)

2. Calderón, A.P., Zygmund, A.: On singular integral. Am. J. Math. 78, 289-309 (1956)

3. Coifman, R.R., Weiss, G.: Extensions of Hardy spaces and their use in analysis. Bull. Am. Math. Soc. 83, 569-645 (1977)

4. Connett, W.C.: Singular integrals near L'1. In: Harmonic Analysis in Euclidean Spaces (Proc. Sympos. Pure Math., Williams Coll., Williamstown, Mass., 1978), Part 1, pp. 163-165. Am. Math. Soc., Providence (1979)

5. Seeger, A.: Singular integral operators with rough convolution kernels. J. Am. Math. Soc. 9, 95-105 (1996)

6. Tao, T.: The weak type $(1,1)$ of $L \log L$ homogeneous convolution operators. Indiana Univ. Math. J. 48, 1547-1584 (1999)

7. Liu, F.: Rough singular integrals associated to surfaces of revolution on Triebel-Lizorkin spaces. Rocky Mt. J. Math. 47(5), 1617-1653 (2017)

8. Liu, F.: Boundedness and continuity of maximal operators associated to polynomial compound curves on Triebel-Lizorkin spaces. Math. Inequal. Appl. 22(1), 25-44 (2019)

9. Liu, F.: A note on Marcinkiewicz integrals associated to surfaces of revolution. J. Aust. Math. Soc. 104, 380-402 (2018)

10. Liu, F., Mao, S., Wu, H.: On rough singular integrals related to homogeneous mappings. Collect. Math. 67(1), 113-132 (2016)

11. Fan, D., Pan, Y.: Singular integral operators with rough kernels supported by subvarieties. Am. J. Math. 119(4), 799-839 (1997)

12. Liu, F.: Integral operators of Marcinkiewicz type on Triebel-Lizorkin spaces. Math. Nachr. 290(1), $75-96$ (2017)

13. Liu, F., Wu, H.: Singular integrals related to homogeneous mappings in Triebel-Lizorkin spaces. J. Math. Inequal. 11(4), 1075-1097 (2017)

14. Liu, F., Xue, Q., Yabuta, K.: Rough maximal singular integral and maximal operators supported by subvarieties on Triebel-Lizorkin spaces. Nonlinear Anal. TMA 171, 41-72 (2018)

15. Liu, F., Xue, Q., Yabuta, K.: Boundedness and continuity of maximal singular integrals and maximal functions on Triebel-Lizorkin spaces. Sci. China Math. To appear. https://doi.org/10.1007/s11425-017-9416-5

16. Cho, Y., Ozawa, T.: Sobolev inequalities with symmetry. Commun. Contemp. Math. 11(3), 355-365 (2009)

17. D'Ancona, P., Cacciafesta, F.: Endpoint estimates and global existence for the nonlinear Dirac equation with potential. J. Differ. Equ. 254(5), 2233-2260 (2013)

18. Xi, S., Zhu, S.: Blow-up criterion for the $3 \mathrm{D}$ non-resistive compressible magnetohydrodynamic equations. J. Dyn. Differ. Equ. To appear. https://doi.org/10.1007/s10884-019-09756-Z

19. Wang, J., Cheng, H., Liu, H., Wang, Y.: Periodic solution and control optimization of a prey-predator model with two types of harvesting. Adv. Differ. Equ. 2018(41), 1 (2018)

20. Liu, F:: On the Triebel-Lizorkin space boundedness of Marcinkiewicz integrals along compound surfaces. Math. Inequal. Appl. 20(2), 515-535 (2017)

21. Liu, F.: Rough maximal functions supported by subvarieties on Triebel-Lizorkin spaces. Rev. R. Acad. Cienc. Exactas Fís. Nat., Ser. A Mat. 112(2), 593-614 (2018) 
22. Lucà, R.: Regularity criteria with angular integrability for the Navier-Stokes equation. Nonlinear Anal. 105, 24-40 (2014)

23. Liu, F.: Boundedness and continuity of maximal operators associated to polynomial compound curves on Triebel-Lizorkin spaces. Math. Inequal. Appl. 22(1), 25-44 (2019)

24. Liu, F., Fu, Z., Jhang, S.: Boundedness and continuity of Marcinkiewicz integrals associated to homogeneous mappings on Triebel-Lizorkin spaces. Front. Math. China 14(1), 95-122 (2019)

25. Machihara, S., Nakamura, M., Nakanishi, K., Ozawa, T.: Endpoint Strichartz estimates and global solutions for the nonlinear Dirac equation. J. Funct. Anal. 219(1), 1-20 (2005)

26. Ozawa, T., Rogers, K.M.: Sharp Morawetz estimates. J. Anal. Math. 121, 163-175 (2013)

27. Sterbenz, J.: Angular regularity and Strichatz estimates for the wave equation. Int. Math. Res. Not. 4(5), 187-231 (2005)

28. Tao, T.: Spherically averaged endpoint Strichartz estimates for the two-dimensional Schrödinger equation. Commun. Partial Differ. Equ. 25(7-8), 1471-1485 (2000)

29. Córdoba, A.: Singular integrals, maximal functions and Fourier restriction to spheres: the disk multiplier revisited. Adv. Math. 290, 208-235 (2016)

30. D'Ancona, P., Lucà, R.: On the regularity set and angular integrability for the Navier-Stokes equation. Arch. Ration. Mech. Anal. 221, 1255-1284 (2016)

31. Cacciafesta, F., Lucà, R.: Singular integrals with angular integrability. Proc. Am. Math. Soc. 144(8), 3413-3418 (2016)

32. Carbery, A., Hernández, E., Soria, F.: The behaviour on radial functions of maximal operators along arbitrary directions and the Kakeya maximal operator. Tohoku Math. J. (2) 41(4), 647-656 (1989)

33. Grafakos, L.: Classical Fourier Analysis, 2nd edn. Prentice Hall, Upper Saddle River (2003)

\section{Submit your manuscript to a SpringerOpen ${ }^{\circ}$ journal and benefit from:}

- Convenient online submission

Rigorous peer review

Open access: articles freely available online

- High visibility within the field

- Retaining the copyright to your article

Submit your next manuscript at $\gg$ springeropen.com 